$\checkmark$ Research Square
Preprints are preliminary reports that have not undergone peer review.

They should not be considered conclusive, used to inform clinical practice, or referenced by the media as validated information.

\title{
An Ideal Neonatal Congenital Heart Disease Screening Plan by Pulse Oximeter and Auscultation in a Large Single Center
}

\section{Yuqiang Huang}

Linyi Maternal and Child Healthcare Hospital

\section{Shiqing Zhong}

Linyi Maternal and Child Healthcare Hospital

\section{Xianmei Zhang}

Linyi Maternal and Child Healthcare Hospital

\section{Linghui Kong}

Linyi Maternal and Child Healthcare Hospital

\section{Wenli Wu}

Linyi Maternal and Child Healthcare Hospital

\section{Shixia Yue}

Linyi Maternal and Child Healthcare Hospital

\section{Ning Tian}

Linyi Maternal and Child Healthcare Hospital

\section{Guanghua Zhu}

Linyi Maternal and Child Healthcare Hospital

\section{Aiqin $\mathrm{Hu}$}

Linyi Maternal and Child Healthcare Hospital Juan Xu

Linyi Maternal and Child Healthcare Hospital Haijan Zhu

Linyi Maternal and Child Healthcare Hospital

\section{Airong Sun}

Linyi Maternal and Child Healthcare Hospital

\section{Fangling Qin}

Linyi Maternal and Child Healthcare Hospital

\section{Ziwen Wang}

Linyi Maternal and Child Healthcare Hospital

\section{Shiqiang Wu ( $D$ 604287252@qq.com )}




\section{Research Article}

Keywords: Congenital heart disease, pulse oximetry, auscultation, screening, critical congenital heart disease

Posted Date: March 2nd, 2022

DOI: https://doi.org/10.21203/rs.3.rs-1384267/v1

License: (c) (i) This work is licensed under a Creative Commons Attribution 4.0 International License. Read Full License 


\section{Abstract \\ Purpose}

To investigate the feasibility and reliability of pulse oximeter (POX) as a fast-screening device for congenital heart disease (CHD) through comparing performances of cardiac auscultation and POX in a large retrospective single center study.

\section{Methods}

Every newborn received auscultation and POX tests separately. When an abnormal murmur or $\mathrm{SpO}_{2}$ level is detected, an echocardiogram will be ordered to confirm the diagnosis of CHD in the newborn.

\section{Results}

From January 1, 2018 to December 31, 2019, there were 44,147 livebirths at the studied hospital where 498 newborns were diagnosed with CHD: 27 newborns by POX screening and 471 by cardiac auscultation. The diagnosis was further confirmed in 458 neonates through echocardiogram. This result puts forth an overall diagnosis rate of $91.97 \%$. Cardiac auscultation detected the majority of CHD cases 438 (95.6\%) while POX only screened 20 (4.4\%) cases. Interestingly, no CHD case was detected by both auscultation examination and POX screening. Auscultation detected most of the common types of CHD, but POX excelled in identifying rare and critical cases. POX screening alone had a very low accuracy of $3.41 \%$ in positive predict value (PPV). On the other hand, auscultation can function well in terms of PPV and negative predict value (NPV) (92.99\% and $99.95 \%$, respectively), but the addition of POX could improve the overall screening performance and reach NPV to $100 \%$. We also validate the finding with the data six months after the study period.

\section{Conclusion}

Auscultation screening operates well in detecting common CHDs whereas POX screening excels in distinguishing rare, yet critical, cases of CHDs. Therefore, an ideal CHD screening plan should combine cardiac auscultation and POX screening.

\section{What Is Known}

- Pulse-oximetry is safe, acceptable, non-invasive and effective.

- Pulse oximetry screening increases early diagnosis of major CHD as well as other important pathology with a very low false positive rate and minimal requirement for extra echocardiograms. 
- Pulse-oximetry plus cardiac auscultation significantly improved the detection rate of major CHD in the early neonatal stage, with high sensitivity and a reasonable false-positive rate.

\section{What is new}

- No CHD case was detected by both auscultation examination and Pulse-oximetry screening.

- Auscultation can function well in terms of positive predict value and negative predict value $(92.99 \%$ and $99.95 \%$, respectively), but the addition of POX could improve the overall screening performance and reach NPV to $100 \%$.

\section{Introduction}

Congenital heart disease (CHD) is the most common type of congenital malformations, with an overall prevalence of $6-10 \%$ in newborns (among which 2-3\%o cases are considered severe). [1] About a quarter of CHD patients require surgery or catheterization in neonatal period, or early infancy. [2] Timely diagnosis and treatment can greatly improve the prognosis of patients. [3] Conversely, delayed diagnosis often leads to serious hypoxia, shock, acidosis, pneumonia, and other complications including death. Studies have reported that delayed diagnosis of critical congenital heart disease (CCHD) led to death in 12 of every 100,000 live births in the United States. [4] Prenatal obstetric ultrasound can detect about $30 \%$ fetuses with $\mathrm{CHD}$ [5], but early neonatal diagnosis is still a big challenge.

Although echocardiography is the gold standard to validate cases of CHD, it usually takes more than 10 minutes to perform. Hence, performing echocardiography for every newborn in areas with limited resources is not practical. As an alternative, pulse oximetry (POX) is easy to operate and requires only 2 to 3 minutes to analyze the results. Besides, POX as an adjunct to current routine practice is likely to be a cost-effective strategy in the light of currently accepted thresholds. [6]

It is highly specific in detecting CCHD with moderate sensitivity, and had been widely employed.[7] Since Dr. Guoying Huang introduced and established CHD screening system via POX to China in 2018, it has been adopted in nationwide clinical routines. [8]

As a large maternal and child healthcare facility, the hospital of study delivers more than 20,000 babies annually. Consequently, offering quick, convenient, and accurate screening tools for CHD detection among newborns are crucial for the local community in the absence of echocardiologist. This health center has combined auscultation and POX in CHD diagnostic tests among newborns since 2018. When babies are recognized with CHD by POX or auscultation, an echocardiography will be requested for confirmation. This study aims to compare and report the performances of auscultation and POX screenings for the first time.

\section{Methods}

\subsection{Study design and participants}


We conducted this retrospective study; from January 1, 2018 to December 31, 2019 at Linyi Maternal and Child Healthcare Hospital, where more than 20,000 infants were delivered annually, to confirm the applicability and accuracy of pulse oximetry combined cardio-auscultation in determining congenital heart disease among neonates.

All consecutive newborns were eligible, irrespective of gestational age, NICU admission, symptom presence or prenatal diagnosis.

This study was approved by institutional ethical review board of Linyi Maternal and Child Healthcare Hospital. Verbal, informed consent was obtained from the participants' parents. The registry number is NCT05105880 (https://register.clinicaltrials.gov).

\subsection{Procedures}

\subsubsection{Cardio-auscultation}

Pediatricians from Departments of Neonatal Medicine and Developmental Medicine are responsible for auscultation screening. A standard pediatric stethoscope (Model 3200, 3M Littmann) was used. Cardiac auscultation was performed on the five points of auscultation (pulmonic, aorta, Erb's point, tricuspid, and mitral) for each newborn under typical conditions of the general maternity ward, and the duration lasted 60 seconds per neonate. When abnormal murmur occurred, an echocardiography would be requested to confirm if the newborn has CHD or not.

\subsubsection{Pulse oximetry}

Physicians from obstetrics department and nursing staff from neonatal department examined POX measurements using a pulse oximeter (Masimo, Irvine, CA, USA), with a multisite reusable or disposable sensor (Model 2329, Masimo). All babies should be screened within 72 hours after birth. Pulse oximeter oxygen saturation ( $\mathrm{SpO2}$ ) was measured from the right hand and on either foot. $\mathrm{SpO}_{2}$ levels is recorded on the nursing report sheet. If the measurement was less than $90 \%$ or the difference between two extremities was more than $3 \%$, the baby would be referred for echocardiography immediately. If one extremity's measurement ranged between $90 \%$ and $94 \%$, the clinician would repeat the test on the baby 4 hours later. If measurements on both extremities were between $90 \%$ and $94 \%$, the baby would be referred for echocardiography.

\subsubsection{Echocardiography}

Physicians from Department of Ultrasonography confirmed the diagnosis of CHD among newborns diagnosed by auscultation or POX through directing echocardiography. Pediatricians then refer the CHD results to cardiology center. On the basis of the severity classification of congenital heart disease recommended by Hoffman's method, we divided cases of the disease into severe, moderate and mild groups, and prescribed treatment plans accordingly. [9] 


\section{Results}

From January 1, 2018 to December 31, 2019, there were 44,147 livebirths in the studied hospital. The demographic characteristics of the screened population were summarized in Table 1. The majority of the newborns were full term births delivered between 37 and 40 gestational weeks, with a median gestational age of 39 weeks. The median birth weight for newborn is $3,420 \mathrm{~g}$, which was consistent with the gestational age of the mother. Three quarters of the babies underwent screening within 24 hours after birth and cesarean section rate was $45.2 \%$. As the study center undertakes the child healthcare in the study region, there is no additional CHD case found among these infants up to 2022.

There were 498 newborns diagnosed with CHD initially - 27 by POX screening and 471 by cardiac auscultation - with an overall diagnostic rate of $1.13 \%$ among all 44,147 livebirths. Of these cases, 458 neonates were confirmed by echocardiography, making the overall diagnostic rate of $\mathrm{CHD}$ via echocardiogram $91.97 \%$ (including 253 male babies and 245 female babies). The screen results are presented in Table 2. The most common types of CHD were PDA (34.3\%), ASD (20.5\%), VSD (8.3\%), and combined complications (34.5\%). Rare CHD included COA, CTA PAPVC, TAPVC, TGA, and TOF, accounting for a total of $2.2 \%$ cases. Mild CHD accounted for $74 \%$ of all cases, followed by moderate CHD (15.3\%), and severe $\mathrm{CHD}(10.7 \%)$. Among all CHD cases, 37 cases were already identified by prenatal screening with ultrasonography.

Of the 458 confirmed CHD subjects, cardiac auscultation alone detected majority of CHD cases 438 (95.6\%) and POX only screened 20 (4.4\%) cases. However, not a single incidence of CHD is recognized by both auscultation and POX screening (Table 3). A potential explanation for this result is that auscultation detected most of the common types of CHD neonates, but POX detected the rare, yet critical CHD types, including COA, CTA, PAPVC, TAPVC, TGA, and TOF. The same observation was made in a new set of data from 2020.

POX screening alone has a very low accuracy of $3.41 \%$ in positive predict value (PPV). Auscultation alone can function well in terms of PPV and negative predict value (NPV) (92.99\% and 99.95\%, respectively), but the addition of POX could improve the overall screening performance and reach NPV to $100 \%$ (Table 4).

As PDA and ASD were not included in prenatal screening by ultrasonography, they were excluded when tracing back prenatal ultrasound screening. Of the remaining 108 cases, 37 fetuses were diagnosed with CHD, including 35 cases of VSD, 1 case of COA, and 1 case of TOF (Table 5).

\section{Discussion}

In this large single-center retrospective study, we applied combined POX and auscultation in routine CHD screening for all newborn infants within 24 hoursafter delivery. Within a two-year period, we identified 458 CHD neonates from 44,147 live births, of which $74 \%$ neonates are diagnosed with mild CHD, $15.3 \%$ with moderate CHD, and $10.7 \%$ with major CHD. Although POX can not detect slight CHD accurately, this 
inexpensive and quick screening technique in conjunction with clinical auscultation can result in a high detection rate of serious CHD.

The most intriguing finding in this screening was that there was no overlap of CHD spectrums between POX detection and auscultation detection. In contrast to common CHDs detected by auscultation, POXdetected CHDs were rare and critical, including COA, CTA, PAPVC, TAPVC, TGA, and TOF, which were consistent with the primary and second target lesions presenting at least mild hypoxemia during the neonatal period.[10] This observation is quite different from two previous research with significant overlaps between two screening techniques. $[8,11]$ The dynamic changes of pulmonary arterial pressure in newborns in combination of all screenings conducted within the first 24 hours after delivery likely contributed to the difference between our observation and the observations made by previous researches. In fetal period, pulmonary circulation was not in use. Instead, it is occupied by amniotic fluid and full of pressure. After birth, because postnatal circulation requires inhalation of air, lung aeration initiates and clears the airway fluid, accompanied by decreasing pulmonary arterial pressure. The pressure is 60 $\mathrm{mmHg}$ at birth and drops to about $30 \mathrm{mmHg}$ at 24 hours of age. [12-14] Since all of the screenings were conducted within the first 24 hours of delivery, which was different from the 72-hour window used in previous studies, it might be difficult to detect abnormal heart murmur when pulmonary arterial pressure still maintained relatively high. Moreover, in one of two previous studies with a large population of 167,190 asymptomatic newborn infants, most POX-recognizable cases of CHD were only detected via auscultation.[11] Considering this current, early-stage, multicenter study investigated the accuracy and feasibility of implementing cardiac auscultation and POX as a screening method in China, there might be targe variations in the POX measurement among different hospitals studied for different researches, resulting in low detection rate by POX in other studies. This study's finding manifests the complementary aspects of POX and auscultation in preliminary diagnosis of CHD among newborns, shedding light on the effectiveness of combined use of both methods in comprehensive neonatal screening, especially regions lacking echocardiologists.

Meanwhile, the commonality of certain types of CHD was different: PDA, VSD, and ASD accounting for $97.6 \%$ of total CHD cases in our study and $\sim 91.1 \%$ in previous studies. The difference in incidence rate and the CHD spectrum could be explained by differences in prenatal management among different hospitals. Tertiary referral hospitals have more medical resources and better prenatal screening and management systems. Pregnant women registered at these hospitals usually received additional prenatal ultrasound screening, which can increase the chance of prenatal diagnosis of major CHD, usually followed with termination of pregnancy. Contrasting to previous multicenter researches which collected data from a wide range of hospitals, our study was conducted at a single, tertiary referral hospital which had better prenatal management systems, prenatal screenings and adequately trained medical staffs.[15]

Some mild congenital defects, including small muscular VSD, ASD and PDA, would become less noticeable or even close spontaneously without intervention.[16] Owing to the natural closure, these neonates should not be included when calculating CHD incidence, or if counted, it will increase the incidence rate at different stages. Moreover, due to limited resources, our study only considered 
observable CHD cases before discharge, whereas other studies offered follow up statistics among neonates after discharge. Thus, previously misdiagnosed or undiagnosed neonates would be determined to have $\mathrm{CHD}$ and added to the total CHD incidence later in the study. Furthermore, some studies would exclude CHD cases that were screened out in prenatal ultrasonography and confirmed in postnatal echocardiography, and symptomatic CHD neonates such as cyanotic subjects would not be included, either. As a whole, these limitations explain the observable differences in overall CHD occurrence and the major type of diagnosed CHD between our study and the previous studies.

\section{Conclusions}

Our study demonstrates that within the first 24 hours after birth, auscultation screening functions well in detecting common CHDs, whereas POX screening excels in differentiating rare and critical CHDs. When combined in CHD screening, these two techniques can generate quick and accurate outcomes in an economic way, which can benefit facilities in low-income area and hospitals short of ward resource. More cohort studies will be established for these infants.

\section{Abbreviations}

ASD: atrial septal defect

CCHD: critical congenital heart disease

CHD: congenital heart disease

COA: coarctation of the aorta

CTA: cor triatriatum

NPV: negative predict value

PAPVC: partial anomalous pulmonary venous connection

PDA: patent ductus arteriosus

POX: pulse oximeter

PPV: positive predict value

Sp02: oxygen saturation

TAPVC: total anomalous pulmonary venous connection

TGA: transposition of the great arteries

TOF: tetralogy of Fallot 
VSD: ventricular septal defect

\section{Declarations}

Funding: This research was supported by the grant from Linyi Maternal and Child Healthcare Hospital.

Conflicts of interest: The authors declare that the research was conducted in the absence of any commercial or financial relationships that could be construed as a potential conflict of interest.

Availability of data and material: Not available.

Code availability: Not applicable.

Authors' contributions: Yuqiang Huang designed the study and drafted the manuscript; Shiqiang Wu, Shiqiang Zhong, Ziwen Wang conducted auscultation and collected data; Xianmei Zhang, Linghui Kong undertook echocardiography; Wenli Wu, Shixia Yue, Ning Tian, Guanghua Zhu, Aiqin Hu, Juan Xu, Haijian Zhu, Airong Sun, Fangling Qin undertook pulse oximeter tests.

Ethics approval: This study was approved by institutional ethical review board of Linyi Maternal and Child Healthcare Hospital. Verbal, informed consent was obtained from the participants' parents. The registry number is NCT05105880 (https://register.clinicaltrials.gov).

Consent to participate: Not applicable.

Consent for publication: This manuscript has not been published and is not under consideration for publication elsewhere. All authors agree to publish on the European Journal of Pediatrics.

Acknowledgments

We would like to thank Zhaoxi Wang, Yinrui Zou, Qin Gao from Harvard International Healthcare Innovation Collaboration Initiatives for helpful discussions and for reviewing our manuscript.

\section{References}

1. <Report on birth defect prevention in China.pdf>.

2. Mahle WT, Newburger JW, Matherne GP, Smith FC, Hoke TR, Koppel R, Gidding SS, Beekman RH, 3rd, Grosse SD: Role of pulse oximetry in examining newborns for congenital heart disease: a scientific statement from the American Heart Association and American Academy of Pediatrics. Circulation 2009, 120(5):447-458.

3. Mellander $\mathrm{M}$, Sunnegårdh J: Failure to diagnose critical heart malformations in newborns before discharge-an increasing problem? Acta Paediatr 2006, 95(4):407-413.

4. Oakley JL, Soni NB, Wilson D, Sen S: Effectiveness of pulse-oximetry in addition to routine neonatal examination in detection of congenital heart disease in asymptomatic newborns. J Matern Fetal 
Neonatal Med 2015, 28(14):1736-1739.

5. Donofrio MT, Moon-Grady AJ, Hornberger LK, Copel JA, Sklansky MS, Abuhamad A, Cuneo BF, Huhta JC, Jonas RA, Krishnan A et al: Diagnosis and treatment of fetal cardiac disease: a scientific statement from the American Heart Association. Circulation 2014, 129(21):2183-2242.

6. Roberts TE, Barton PM, Auguste PE, Middleton LJ, Furmston AT, Ewer AK: Pulse oximetry as a screening test for congenital heart defects in newborn infants: a cost-effectiveness analysis. Arch Dis Child 2012, 97(3):221-226.

7. Thangaratinam S, Brown K, Zamora J, Khan KS, Ewer AK: Pulse oximetry screening for critical congenital heart defects in asymptomatic newborn babies: a systematic review and meta-analysis. Lancet 2012, 379(9835):2459-2464.

8. Zhao Q-m, Ma X-j, Ge X-I, Liu F, Yan W-I, Wu L, Ye M, Liang X-c, Zhang J, Gao Y et al: Pulse oximetry with clinical assessment to screen for congenital heart disease in neonates in China: a prospective study. The Lancet 2014, 384(9945):747-754.

9. <The Incidence of Congenital Heart Disease.pdf>.

10. Engel MS, Kochilas LK: Pulse oximetry screening: a review of diagnosing critical congenital heart disease in newborns. Med Devices (Auckl) 2016, 9:199-203.

11. Hu XJ, Ma XJ, Zhao QM, Yan WL, Ge XL, Jia B, Liu F, Wu L, Ye M, Liang XC et al: Pulse Oximetry and Auscultation for Congenital Heart Disease Detection. Pediatrics 2017, 140(4).

12. Hu Q, Ren WD, Mao J, Li J, Qiao W, Bi WJ, Xiao YJ, Zhan Y, Xu M, Liu CX et al: Changes in pulmonary artery pressure during early transitional circulation in healthy full-term newborns. Ultrasonics 2015, 56:524-529.

13. Kang C, Zhao E, Zhou Y, Zhao H, Liu Y, Gao N, Huang X, Liu B: Dynamic Changes of Pulmonary Arterial Pressure and Ductus Arteriosus in Human Newborns From Birth to 72 Hours of Age. Medicine (Baltimore) 2016, 95(3):e2599.

14. Greenough A, Khetriwal B: Pulmonary hypertension in the newborn. Paediatr Respir Rev 2005, 6(2):111-116.

15. Zhao QM, Niu C, Liu F, Wu L, Ma XJ, Huang GY: Accuracy of cardiac auscultation in detection of neonatal congenital heart disease by general paediatricians. Cardiol Young 2019, 29(5):679-683.

16. Sadowski SL: Congenital cardiac disease in the newborn infant: past, present, and future. Crit Care Nurs Clin North Am 2009, 21(1):37-48, vi.

\section{Tables}

Table 1. Demographic characteristic of newborns 
Variables

Total

Normal

Mild CHD

Moderate

Severe CHD

$\mathrm{N}=44,147$

$N=43,689$

$\mathrm{N}=339$

CHD

$\mathrm{N}=70$

Gestational age

(weeks)

\begin{tabular}{llllll}
\hline$<37$ & $2,691(6.1 \%)$ & $2,668(6.1 \%)$ & $14(4.1 \%)$ & $6(8.6 \%)$ & $3(6.1 \%)$ \\
\hline $37-40$ & $\begin{array}{l}37,229 \\
(84.3 \%)\end{array}$ & $\begin{array}{l}36,841 \\
(84.3 \%)\end{array}$ & $290(85.6 \%)$ & $57(81.4 \%)$ & $41(83.7 \%)$ \\
\hline$>40$ & $4,227(9.6 \%)$ & $4,180(9.6 \%)$ & $35(10.3 \%)$ & $7(10.0 \%)$ & $5(10.2)$ \\
\hline $\begin{array}{l}\text { Gestational age } \\
\text { (weeks) }\end{array}$ & $39(38-40)$ & $39(38-40)$ & $39(39-40)$ & $39(38-39)$ & $39(39-40)$ \\
\hline Birth Weight (g) & $3,420(3,130-$ & $3,420(3,130-$ & $3480(3170-$ & $3245(3000-$ & $3440(3160-$ \\
& $3,720)$ & $3,720)$ & $3800)$ & $3515)$ & $3790)$ \\
\hline $\begin{array}{l}\text { Age at screening } \\
\text { (hours) }\end{array}$ & $20(15.0-$ & $20(15.0-$ & $20(16.0-$ & $19.5(15.8-$ & $19(15.5-$ \\
\hline Delivery method & $24.0)$ & $24.0)$ & $24.0)$ & $24.0)$ & $24.0)$ \\
\hline Natural birth & 24,159 & 23,894 & $205(60.4 \%)$ & $35(50.0 \%)$ & $25(51.0 \%)$ \\
\hline Cesarean section & $\begin{array}{l}(54.7 \%) \\
(4,946\end{array}$ & $\begin{array}{l}19,754 \\
(45.2 \%)\end{array}$ & $133(39.2 \%)$ & $35(50.0 \%)$ & $24(49.0 \%)$ \\
\hline $\begin{array}{l}\text { Natural birth to } \\
\text { cesarean section }\end{array}$ & $42(0.1 \%)$ & $41(0.1 \%)$ & $1(0.2 \%)$ & 0 & 0 \\
\hline
\end{tabular}

Table 2. Result of CHD cases 


\begin{tabular}{|c|c|c|c|c|}
\hline \multirow[t]{2}{*}{ CHD Type } & \multirow{2}{*}{$\begin{array}{l}\text { Mild CHD } \\
\text { Case counts } \\
(\%)\end{array}$} & \multirow{2}{*}{$\begin{array}{l}\text { Moderate CHD } \\
\text { Case counts (\%) }\end{array}$} & \multicolumn{2}{|l|}{ Severe CHD } \\
\hline & & & Case counts (\%) & $\begin{array}{l}\text { Total case counts } \\
(\%)\end{array}$ \\
\hline ASD & 70ه15.3ه & $24 \otimes 5.2 \rrbracket$ & OखOQ & $94(20.5)$ \\
\hline ASD\&PDA & 76ه16.6ه & $13 \rrbracket 2.8 \rrbracket$ & 10区2.2区 & $99(21.6)$ \\
\hline $\mathrm{COA}$ & 0 & $1 \otimes 0.2 \rrbracket$ & 0 & $1(0.2)$ \\
\hline CTA & 0 & $1 \otimes 0.2 \rrbracket$ & 0 & $1(0.2)$ \\
\hline PAPVC & 0 & $2 \rrbracket 0.4 \rrbracket$ & 0 & $2(0.4)$ \\
\hline PDA & 127凶27.7凶 & $9 \llbracket 2.0 \rrbracket$ & 21ه4.6区 & $157(34.3)$ \\
\hline TAPVC & 0 & 0 & $3 \rrbracket 0.7 \rrbracket$ & $3(0.6)$ \\
\hline TGA & 0 & 0 & $2 \varangle 0.4 \rrbracket$ & $2(0.4)$ \\
\hline TOF & 0 & 0 & $2 \varangle 0.4 \rrbracket$ & $2(0.4)$ \\
\hline VSD & $27 \rrbracket 5.9 \rrbracket$ & $7 \rrbracket 1.5 \rrbracket$ & $4 \llbracket 0.9 \rrbracket$ & $38(8.3)$ \\
\hline VSD\&ASD & $14 \llbracket 3.1 \rrbracket$ & $5 \bigotimes 1.1 \rrbracket$ & $2 \bigotimes 0.4 \rrbracket$ & $21(4.6)$ \\
\hline VSD\&ASD\&PDA & $9 \llbracket 2.0 \rrbracket$ & $3 \llbracket 0.7 \rrbracket$ & $2 \bigotimes 0.4 \rrbracket$ & $14(3.1)$ \\
\hline VSD\&PDA & 16ه3.5区 & $5 \otimes 1.1 \rrbracket$ & $3 \otimes 0.7 \rrbracket$ & $24(5.2)$ \\
\hline Total & $339 \otimes 74.0 \rrbracket$ & 70ه15.3ه & 49ه10.7凶 & $458(100)$ \\
\hline
\end{tabular}

Table 3. Detection rate for CHD types 
Detection rate

\begin{tabular}{|c|c|c|}
\hline AD types & $\mathrm{N}$ & $\begin{array}{l}\text { Auscultation } \\
\text { alone }\end{array}$ \\
\hline
\end{tabular}

\section{Study Period Data}

\begin{tabular}{lllll}
\hline ASD & 94 & 94 & 0 & 0 \\
\hline ASD\&PDA & 99 & 99 & 0 & 0 \\
\hline COA & 1 & 0 & 1 & 0 \\
\hline CTA & 1 & 0 & 1 & 0 \\
\hline PAPVC & 2 & 0 & 2 & 0 \\
\hline PDA & 157 & 154 & 3 & 0 \\
\hline TAPVC & 3 & 0 & 3 & 0 \\
\hline TGA & 2 & 0 & 2 & 0 \\
\hline TOF & 2 & 0 & 2 & 0 \\
\hline VSD & 38 & 35 & 3 & 0 \\
\hline VSD\&ASD & 21 & 20 & 1 & 0 \\
\hline VSD\&ASD\&PDA & 14 & 12 & 2 & 0 \\
\hline VSD\&PDA & 24 & 24 & 0 & 0
\end{tabular}

\section{First Half of 2020}

Data

\begin{tabular}{lllll}
\hline ASD & 6 & 6 & 0 & 0 \\
\hline VSD & 8 & 8 & 0 & 0 \\
\hline PDA & 10 & 10 & 0 & 0 \\
\hline ASD\&VSD & 2 & 2 & 0 & 0 \\
\hline VSD\&PDA & 2 & 2 & 0 & 0 \\
\hline TOF & 1 & 0 & 1 & 0 \\
\hline TAPVC & 1 & 0 & 1 & 0 \\
\hline In total & 30 & 28 & 2 & 0
\end{tabular}

Table 4. Screening accuracy for CHD new born $(n=44,147)$ 


\begin{tabular}{llll} 
& $\begin{array}{l}\text { Pulse oximetry } \\
\text { alone }\end{array}$ & $\begin{array}{l}\text { Auscultation } \\
\text { alone }\end{array}$ & $\begin{array}{l}\text { Auscultation or pulse } \\
\text { oximetry }\end{array}$ \\
\hline True positives & 31 & 438 & 458 \\
\hline False negatives & 427 & 20 & 0 \\
\hline False positives & 877 & 33 & 40 \\
\hline True negatives & 42,812 & 43,656 & 43,649 \\
\hline Sensitivity & $6.77 \%$ & $95.63 \%$ & $100.00 \%$ \\
\hline Specificity & $97.99 \%$ & $99.92 \%$ & $99.91 \%$ \\
\hline Positive Predict Value & $3.41 \%$ & $92.99 \%$ & $91.97 \%$ \\
\hline $\begin{array}{l}\text { Negative Predict } \\
\text { Value }\end{array}$ & $99.01 \%$ & $99.95 \%$ & $100 \%$
\end{tabular}

Table 5. Prenatal trace back of confirmed CHD cases excluding PDA and ASD cases $(n=108)$

\begin{tabular}{lll} 
Conditions & Case counts & Percentage (\%) \\
\hline Prenatal identification of CHD & & \\
\hline VSD & 35 & 32.41 \\
\hline COA & 1 & 0.93 \\
\hline TOF & 1 & 0.93 \\
\hline Prenatal identification of non-CHD & 51 & 47.22 \\
\hline Unclear ultrasound image & 3 & 2.78 \\
\hline No prenatal screening & 14 & 12.96 \\
\hline Lost to trace back & 3 & 2.78
\end{tabular}

\section{Figures}




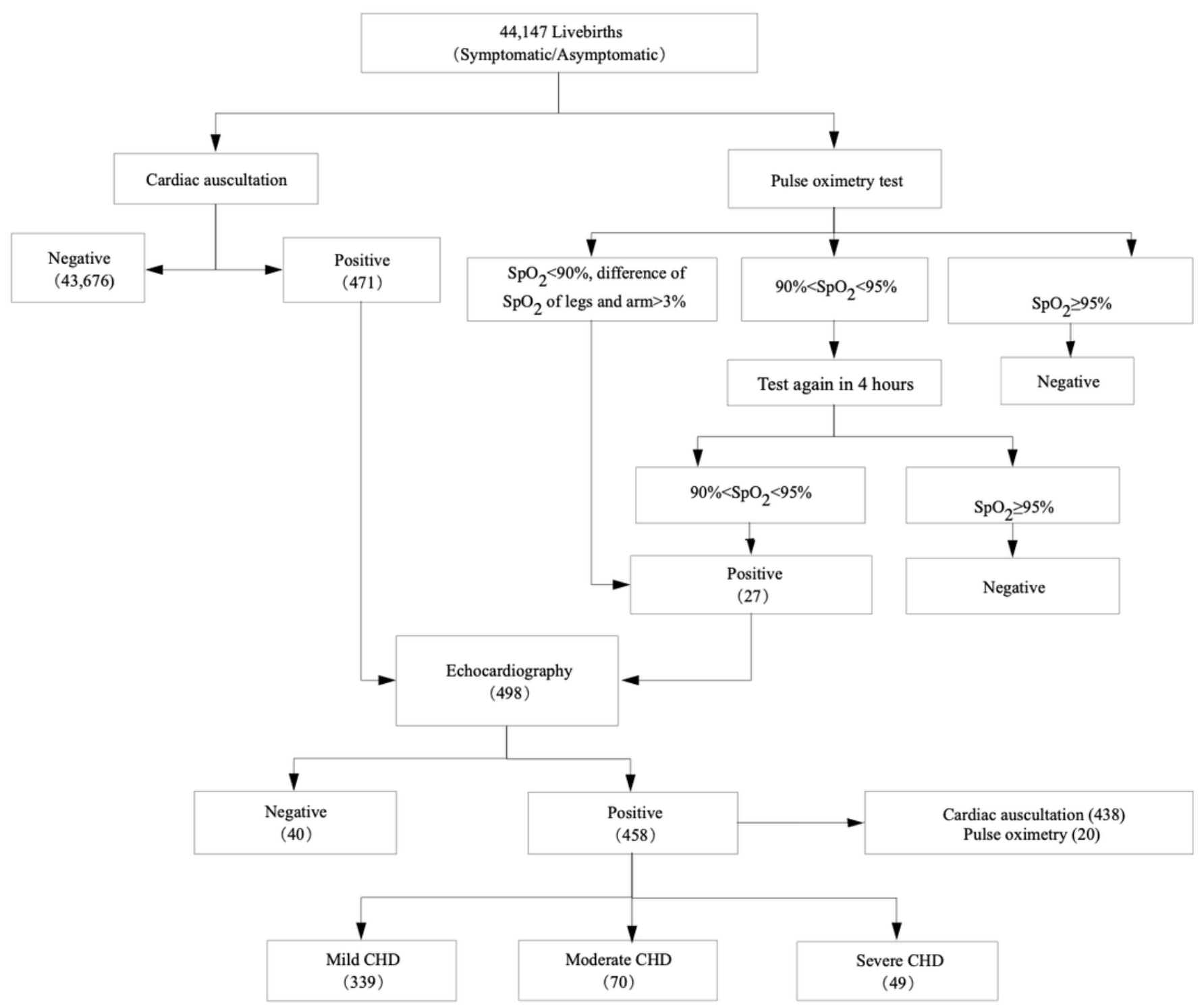

Figure 1

Screening process 